\title{
In silico Analysis on hERG Channel Blocking Effect of a Series of T-type Calcium Channel Blockers
}

\author{
Jae Wan Jang, ${ }^{\dagger,}$ Chi Man Song, ${ }^{\dagger}$ Kee Hyun Choi, ${ }^{\dagger}$ Yong Seo Cho, ${ }^{\dagger,}$ Du-Jong Baek, ${ }^{\dagger}$ Kye Jung Shin, ${ }^{\dagger}$ and Ae Nim Pae ${ }^{\dagger, \S, *}$ \\ ${ }^{\dagger}$ Life \& Health Division, Korea Institute of Science and Technology, PO Box 131, Cheongryang, Seoul 130-650, Korea \\ *E-mail: anpae@kist.re.kr \\ ${ }^{\ddagger}$ Department of Chemistry, College of Natural Sciences, Sangmyung University, Seoul 110-743, Korea \\ ${ }^{\S}$ Department of Medicinal \& Pharmaceutical Chemistry, School of Science, University of Science and Technology, \\ Daejeon 305-333, Korea \\ Received November 2, 2010, Accepted November 15, 2010
}

\begin{abstract}
Human ether-a-go-go related gene (hERG) potassium channel blockade, an undesirable side effect which might cause sudden cardiac death, is one of the major concerns facing the pharmaceutical industry. The purpose of this study is to develop an in silico QSAR model which uncovers the structural parameters of T-type calcium channel blockers to reduce hERG blockade. Comparative molecular similarity indices analysis (CoMSIA) was conducted on a series of piperazine and benzimidazole derivatives bearing methyl 5-(ethyl(methyl)amino)-2-isopropyl-2-phenylpentanoate moieties, which was synthesized by our group. Three different alignment methods were applied to obtain a reliable model: ligand based alignment, pharmacophore based alignment, and receptor guided alignment. The CoMSIA model with receptor guided alignment yielded the best results : $r^{2}=0.955, q^{2}=0.781, r_{\text {pred }}^{2}=0.758$. The generated CoMSIA contour maps using electrostatic, hydrophobic, H-bond donor, and acceptor fields explain well the structural requirements for hERG nonblockers and also correlate with the lipophilic potential map of the hERG channel pore.
\end{abstract}

Key Words: hERG, T-type calcium channel blockers, CoMSIA

\section{Introduction}

Long QT syndrome (LQTS) is a disorder of ventricular repolarization, which is associated with the development of cardiac arrhythmias or the degeneration into sudden cardiac death during a severe case. It is characterized by the prolongation of the QT interval in a body surface electrocardiogram (ECG) which indicates the time between initial depolarization and final repolarization of the ventricles. ${ }^{1}$ Inherited LQTS can be caused by the mutations in the human ether-à-go-go related gene (hERG), which is highly expressed as a potassium channel in the heart system and mediates cardiac repolarization, ${ }^{1-3}$ while acquired LQTS can occur via the blocking of the hERG channel either by the medications for arrhythmia or by common noncardiac medications. ${ }^{1}$ This hERG channel blockade by drugs has resulted in safety concerns by health regulatory authorities and pharmaceutical companies and has become one of the reasons for the withdrawal of many drugs from the market. ${ }^{4}$ Now, testing the hERG blockade is a routine process at an early stage of drug development before clinical testing. ${ }^{5}$

For these reasons, several attempts have been made to find out the binding mechanism of the hERG blockers. Above all, many studies have discovered the hERG channel structure, which has helped researchers understand about drug binding. Although still no crystal structure of human has been available so far, the crystal structure of mammalian potassium channel $(\mathrm{Kv} 1.2)^{6}$ and the bacterial $\mathrm{K}+$ channel $\left(\mathrm{KcsA},{ }^{7} \mathrm{MthK},{ }^{8}\right.$ and $\mathrm{KvAP}^{9}$ ) have given us an useful insight into the structural information of hERG channel. It is formed by the coassembly of four subunits, each of which is constructed by six $\alpha$-helical transmembrane domains, designated as S1 to S6 including pore helix region between S5 and S6. Among them, S6 and the pore helix cover the drug binding site. Ala-scanning mutagenesis studies have identified critical residues interacting with drugs on those transmembranes. ${ }^{5}$ Those are two aromatic residues and two polar residues : Tyr 652 and Phe 656 on S6 and Thr 623 and Ser 624 on the pore helix. ${ }^{5}$ Some residues are also known as interacting with hERG blockers by the indirect method through an allosteric effect. ${ }^{5}$ On the other hand, it was also found that the dimension of the pore also changed according to its conformational alteration : open state and closed state. ${ }^{1}$ The electrophysiology experiments revealed that the channel must be open state for drugs to bind. However, after a drug binds to the pore, S6 can move towards an inner direction, which results in some closing of the inner cavity. This also causes the changing of the binding interaction. Therefore, the closed as well as open state of the hERG channel has been studied together.

In spite of those efforts, the mechanism of the binding of hERG channel blockers still remains doubtful. To make things worse, a structural diversity of hERG blockers leads to confusion and difficulties in understanding this process. ${ }^{10}$ In an effort to solve this complex and multi-faceted problem, several in silico methods have been applied to characterization of the hERG channel blocking property. A ligand based method thatcan be applied when there is no available crystal structure information of the receptor is one of the approaches that has been used so far. There have been various applications regarding a ligand based method about the hERG in the last few years : Classification usng decision tree, ${ }^{11,12}$ support vector machine, ${ }^{13,14,15}$ bayesian, ${ }^{16}$ artificial neural network, ${ }^{17,18}$ and QSAR using two dimentional $^{19,20}$ or three dimentional method by means of pharmacophore, ${ }^{21}$ CoMFA, ${ }^{22}$ CoMSIA,${ }^{23}$ GRIND descriptors. ${ }^{24}$ Recently a similarity-based classifier by topomer search was proposed by Britta Nisius et al. ${ }^{25}$ 
A lot of studies reported so far were hERG in silico model using diverse scaffold from different laboratories because it was difficult to collect many compounds having same scaffold with hERG inhibitory data or because it was needed to utilize diverse scaffold for its unique property of hERG channel. However, studies about hERG using compounds having similar scaffold were also carried out. Pearlstein et al. proposed CoMSIA model based on sertindole and its analogues with some of other hERG blockers.

We have developed T-type calcium channel blockers for the treatment of cardiovascular, neuronal, and endocrine systems. ${ }^{30}$ Many of structurally unique calcium channel blockers have been known to block the hERG channel. ${ }^{66-70}$ Furthermore, our analogues are based on Mibefradil (Posicor ${ }^{\circledR}$, Hoffman-La Roche) (Fig. 10A), which have high hERG blocking activity $\left(\mathrm{IC}_{50}=\right.$ $1.43 \mu \mathrm{M}$, HEK cells), ${ }^{65}$ and other potent analogues. Thus, it makes us more cautious about hERG channel side effect.

In this work, to predict the hERG channel blocking activity of our T-type calcium channel blockers having 5-ethylmethylamino-2-isopropyl-2-phenylpentanoate moieties, we adopted CoMSIA $^{26}$ technique, one of the popular ligand based molecular modeling approaches. Its strong advantage is to offer users graphical representation of structural requirement for the activity. It will be much easier for medicinal chemists to interpret the model shown by graphical representation. To overcome the difficulties of aligning the highly flexible compounds for CoMSIA, diverse alignment strategies were tried and compared to each other. Before generating the model, the binding affinity to hERG channel of all the compounds was assessed by a single hERG electrophysiological assay in our lab and this guaranteed the internal consistency of the data set. We demonstrate that the derived best CoMSIA model using those data sets will give insight into the structural requirements of T-type calcium channel blockers to eliminate the hERG side effects and help us to design novel T-type calcium channel blockers without hERG toxicity.

\section{Computational Methods}

Dataset. A series of 38 T-type calcium channel blockers, which had been already synthesized by our group, ${ }^{30}$ were used as a dataset. The in vitro hERG channel blocking activites of

Table 1. Experimental and predicted hERG blocking activity of benzimidazole derivatives

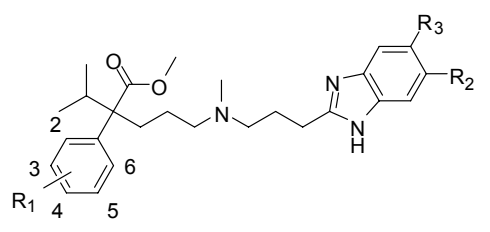

\begin{tabular}{|c|c|c|c|c|c|c|c|}
\hline \multirow{2}{*}{ Compd $^{a}$} & \multirow{2}{*}{$\mathrm{R} 1$} & \multirow{2}{*}{$\mathrm{R} 2$} & \multirow{2}{*}{$\mathrm{R} 3$} & \multirow{2}{*}{ Experimental $\mathrm{pIC}_{50}$} & \multicolumn{3}{|c|}{ Predicted $\mathrm{pIC}_{50}$} \\
\hline & & & & & Alignment 1 & Alignment 2 & Alignment 3 \\
\hline 1 & 4-OMe & $\mathrm{Me}$ & $\mathrm{Me}$ & 5.548 & 5.696 & 5.388 & 5.510 \\
\hline 2 & 6-OMe & $\mathrm{H}$ & $\mathrm{H}$ & 6.678 & 6.439 & 6.554 & 6.649 \\
\hline 3 & 6-OMe & $\mathrm{Me}$ & $\mathrm{Me}$ & 5.607 & 5.744 & 5.559 & 5.657 \\
\hline 4 & 6-OMe & $\mathrm{H}$ & $\mathrm{OMe}$ & 6.201 & 6.482 & 6.448 & 6.238 \\
\hline 5 & 3,4-OMe & $\mathrm{H}$ & $\mathrm{H}$ & 6.161 & 6.087 & 6.134 & 6.145 \\
\hline 6 & $4-\mathrm{F}$ & $\mathrm{H}$ & $\mathrm{H}$ & 6.456 & 6.003 & 6.236 & 6.320 \\
\hline $7 *$ & $4-\mathrm{F}$ & $\mathrm{H}$ & $\mathrm{OMe}$ & 6.509 & 6.071 & 5.836 & 6.352 \\
\hline 8 & $4-\mathrm{F}$ & $\mathrm{Me}$ & $\mathrm{Me}$ & 5.650 & 5.816 & 5.792 & 5.759 \\
\hline 9 & & & & 5.182 & 5.213 & 5.344 & 5.105 \\
\hline 10 & & & & 5.176 & 5.091 & 5.248 & 5.171 \\
\hline 11 & & & & 5.796 & 5.932 & 5.769 & 5.839 \\
\hline
\end{tabular}

\footnotetext{
${ }^{a}$ Compounds labeled with an “*” symbol are the test set.
} 
Table 2. Experimental and predicted hERG blocking activity of piperazine derivatives

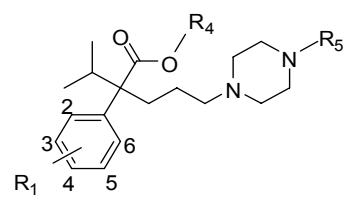

\begin{tabular}{|c|c|c|c|c|c|c|c|}
\hline \multirow{2}{*}{ Compd } & \multirow{2}{*}{$\mathrm{R} 1$} & \multirow{2}{*}{ R4 } & \multirow{2}{*}{ R5 } & \multirow{2}{*}{ Experimental $\mathrm{pIC}_{50}$} & \multicolumn{3}{|c|}{ Predicted $\mathrm{pIC}_{50}$} \\
\hline & & & & & Alignment 1 & Alignment 2 & Alignment 3 \\
\hline 12 & $\mathrm{H}$ & Et & 2-Methoxyphenyl & 5.991 & 5.734 & 6.023 & 5.903 \\
\hline 13 & $\mathrm{H}$ & Et & 3-Methoxyphenyl & 5.728 & 5.656 & 5.684 & 5.793 \\
\hline 14 & $\mathrm{H}$ & Et & 2-Fluorophenyl & 5.387 & 5.471 & 5.440 & 5.363 \\
\hline 15 & $\mathrm{H}$ & Et & 4-Fluorophenyl & 5.367 & 5.623 & 5.520 & 5.449 \\
\hline 16 & $4-\mathrm{Br}$ & $\mathrm{Me}$ & 4-Trifluoromethylbenzyl & 5.684 & 5.720 & 5.685 & 5.679 \\
\hline 17 & $4-\mathrm{Br}$ & $\mathrm{Me}$ & 4-Methoxyphenyl & 5.662 & 5.683 & 5.759 & 5.769 \\
\hline 18 & $4-\mathrm{Br}$ & $\mathrm{Me}$ & 4-Methylbenzyl & 5.830 & 5.467 & 5.589 & 5.747 \\
\hline 19 & $4-\mathrm{Br}$ & $\mathrm{Me}$ & 2-Chloro,6-fluorobenzyl & 5.127 & 5.485 & 5.239 & 5.186 \\
\hline 20 & $5-\mathrm{Br}$ & $\mathrm{Me}$ & 3-Fluorobenzyl & 5.652 & 5.520 & 5.521 & 5.588 \\
\hline $21^{*}$ & $3-\mathrm{Br}$ & $\mathrm{Me}$ & 3,4,5-Trimethoxybenzyl & 5.435 & 5.406 & 5.396 & 5.136 \\
\hline 22 & $3-\mathrm{Br}$ & $\mathrm{Me}$ & 3-Methylbenzyl & 5.254 & 5.277 & 5.190 & 5.252 \\
\hline $23 *$ & $3-\mathrm{Br}$ & $\mathrm{Me}$ & 4-Methylbenzyl & 5.088 & 5.275 & 5.311 & 5.254 \\
\hline 24 & $3-\mathrm{Br}$ & $\mathrm{Me}$ & 4-Chlorobenzyl & 5.250 & 5.349 & 5.272 & 5.185 \\
\hline 25 & $4-\mathrm{F}$ & $\mathrm{Me}$ & Phenyl & 5.815 & 5.714 & 5.712 & 5.689 \\
\hline $26^{*}$ & $4-\mathrm{F}$ & $\mathrm{Me}$ & 6-Fluorophenyl & 5.287 & 5.551 & 5.584 & 5.589 \\
\hline 27 & $4-\mathrm{F}$ & $\mathrm{Me}$ & 3-Fluorobenzyl & 5.580 & 5.707 & 5.549 & 5.590 \\
\hline 28 & $4-\mathrm{F}$ & $\mathrm{Me}$ & 4-Fluorobenzyl & 5.735 & 5.701 & 5.647 & 5.645 \\
\hline 29 & $4-\mathrm{F}$ & $\mathrm{Me}$ & 3-Methoxyphenyl & 5.796 & 5.736 & 5.828 & 5.827 \\
\hline $30 *$ & $4-\mathrm{F}$ & $\mathrm{Me}$ & 4-Methoxyphenyl & 5.889 & 5.726 & 5.722 & 5.634 \\
\hline $31^{*}$ & $4-\mathrm{F}$ & $\mathrm{Me}$ & 4-Methoxybenzyl & 5.708 & 5.636 & 5.750 & 5.625 \\
\hline $32 *$ & $4-\mathrm{F}$ & $\mathrm{Me}$ & 5-Methylbenzyl & 5.611 & 5.611 & 5.588 & 5.678 \\
\hline 33 & $4-\mathrm{F}$ & $\mathrm{Me}$ & 4-Methylbenzyl & 5.456 & 5.526 & 5.615 & 5.680 \\
\hline 34 & $4-\mathrm{F}$ & $\mathrm{Me}$ & 4-Isobutylbenzyl & 5.807 & 5.645 & 5.800 & 5.742 \\
\hline 35 & $4-\mathrm{F}$ & $\mathrm{Me}$ & 4-Chlorobenzyl & 5.435 & 5.599 & 5.578 & 5.612 \\
\hline $36^{*}$ & $4-\mathrm{F}$ & $\mathrm{Me}$ & 3,4-Dichlorobenzyl & 5.678 & 5.644 & 5.523 & 5.616 \\
\hline 37 & & & & 4.909 & 4.768 & 4.813 & 4.888 \\
\hline 38 & & & & 5.068 & 5.103 & 5.051 & 5.011 \\
\hline
\end{tabular}

${ }^{a}$ Compounds labeled with an “*” symbol are the test set.

those compounds were tested with hERG potassium channels expressed in CHO-K1 cells. The in vitro $\mathrm{hERG}$ inhibitory activity $\left(\mathrm{IC}_{50}\right)$ was converted into a $\mathrm{pIC}_{50}\left(-\log \mathrm{IC}_{50}\right)$ value, and used as a dependant variable for the CoMSIA analysis (Table 1, 2). The whole dataset was divided into a training set and a test set in an approximately standard ratio of 4:1 for a QSAR study the training set with 30 compounds, and the test set with 8 compounds. The training set was used to build the QSAR models, while the test set was employed to access the predictive ability of a generated model. The test set was selected in a way that covers the wide range of activity values.

Molecular Modeling and Alignment. All calculations were 
performed using molecular modeling software SYBYL 8.1, 31 installed on a Linux workstation (CentOS 5.2, Intel Core2 Quad Q6700 2.66 GHz) and Maestro 8.0 ${ }^{32}$ installed on a Linux workstation (CentOS 4.7, Dual-Core Opteron Processor 2216). The result of the CoMSIA analysis is strongly dependant on the ligand alignment method. Thus, three different alignments were tested to identify the best alignment approach to our data set.

\section{Scheme 1 : Ligand based alignment}

Simulated annealing was applied to get the ideally good global minimum conformation of the most active compound. ${ }^{33}$ All the other molecules were created from this optimized compound. Structures were drawn from Sketch Molecule in SYBYL 8.1. For a simulated annealing, the system was heated up to a temperature of $700 \mathrm{~K}$ for $1000 \mathrm{fs}$, and then annealed to $200 \mathrm{~K}$ for $1000 \mathrm{fs}$. The annealing function was exponential, and ten such cycles were run. When creating molecules from the template, initial minimization was carried out by aggregating unchanged part of the new compounds using the AGGREGATE module in SYBYL 8.1 and tolerating changed bonds and atoms to minimize. Full minimization followed after that process. Energy minimizations were performed by the Powell method applying a Tripos force field ${ }^{34}$ and a Gasteiger-Marsili ${ }^{35}$ charge. A convergence criterion was set to $0.05 \mathrm{kcal} /(\mathrm{mol} \AA)$, and maximum iteration was at 100 . After generating all the geometry of compounds, the alignment was carried out using the most active compound. To perform the 'Rigid Field Fit' alignment supplied within SYBYL, the steric and electrostatic fields of that compound were extracted for a template. The geometry of the remaining molecules were adjusted to those calculated template fields. The resultant alignment of the training and the test set is shown in Fig. 2A.

\section{Scheme 2: Pharmacophore based alignment}

Pharmacophore generation and data set alignment were performed using PHASE (version 2.5, 2007; Schrödinger, LLC, New York, NY). We drew structures in Maestro interface and optimized them in Ligprep module implemented in Maestro. Those minimized conformations of all the compounds were subjected to the project table in the Develop Pharmacophore Hypothesis panel in Maestro. Diverse conformations of all the compounds were generated by the torsion angle search method ${ }^{36}$ followed by minimization using an OPLS-2005 force field with a solvation treatment as a distance-dependent dielectric. The maximum number of conformers was set to 1000 per structure, and the maximum relative energy difference was set to $10.0 \mathrm{kcal} /$ mol. Compounds having an activity greater than 6.0 of $\mathrm{pIC}_{50}$ was determined as active for evaluating the pharmacophore hypothesis using survival score. The pharmacophore was generated based on the six built-in types of pharmacophore feature provided in PHASE : hydrogen bond acceptor (A), hydrogen bond donor $(\mathrm{D})$, hydrophobe $(\mathrm{H})$, negative ionizable area $(\mathrm{N})$, positive ionizable area $(\mathrm{P})$, and aromatic ring $(\mathrm{R}) .{ }^{36}$ After generating the pharmacophore, those pharmacophore hypotheses were ranked according to survival score to get the best one. The survival score will be high when the pharmacophore hypothesis matches the active compounds well. After that, we selected the best pharmacophore hypothesis based on the statistical result of the 3DQSAR model. Partial least-square regression was conducted to build the 3D-QSAR model. The QSAR module in Phase was used and it employs the information whether or not the ligand occupy the pharmacophore in the space and how well it correlates with its experimental activity value. All molecules mapped to the selected bestpharmacophore hypothesis were imported to the SYBYL interface for CoMSIA analysis. Selected alignment of the training and the test set using the best pharmacophore hypothesis is illustrated in Fig. 2B.

\section{Scheme 3: Receptor guided alignment}

We tried docking of the most active compound and prepared all the rest of molecules based on that conformation. We used the open state MthK derived hERG homology model by Imai et al. ${ }^{10}$ for docking because it is known that many hERG blockers bind to the receptor when it is in the open state. ${ }^{37-42}$ The structure was imported to Maestro, and prepared with the Protein Preparation Wizard. The most reasonable binding conformation of the most active compound was predicted using Glide 3.0 (Schrodinger, LLC, Portland, OR) with extra precision scoring. The rest of the molecules were built with the Maestro build panel based on that structure. Energy minimization of each compound was performed on the MacroModel module in Maestro with an OPLS-2005 force field and a constant dielectric for electrostatic treatment. Because we assumed that the rest of the compounds will occupy the same $3 \mathrm{D}$ position in the binding site as the most active compound $^{43,44}$ ligands were enclosed by the receptor binding site to avoid relaxation of the original docked conformation when doing minimization. The changed part of the structure was allowed to minimize, while a constraint was applied to the atom of the unchanged portion with a force constant of 100 . The enclosing receptor residues were frozen. All compounds minimized were exported to SYBYL to develop the CoMSIA model. The resulting alignment was shown in Fig. 2C.

CoMSIA Analyses. The CoMSIA model was generated using the QSAR module in SYBYL 8.1. Five different similarity fields were generated such as steric, electrostatic, hydrophobic, hydrogen bond donor, and hydrogen bond acceptor. CoMSIA uses a Gaussian function for its calculation of similarity indices, and we used 0.3 , which is thedefault value, for its attenuation factor. These calculations of the CoMSIA similarity indices were on the regularly spaced grid points $(2 \AA)$ surrounding the prealigned molecules. The equation for the similarity indices is as follows:

$$
A_{F, K}^{q}(j)=-\sum_{i=1}^{n} \omega_{p r o b e, k} \omega_{i k} e^{-\alpha r_{i q}^{2}}
$$

where A is the similarity index at the grid point $\mathrm{q}$, summed over all atoms I of the molecule $\mathrm{j}$ under investigations ; $\omega_{\text {probe, } \mathrm{k}}$ is the probe atom; $\omega_{\mathrm{ik}}$ is the actual value of the physicochemical property $\mathrm{k}$ of atom $\mathrm{I} ; \mathrm{r}_{\mathrm{iq}}$ is the mutual distance between the probe atom at grid point $\mathrm{q}$ and the atom $\mathrm{i}$ of the test molecule; $\mathrm{R}$ is the attenuation factor. Region focusing ${ }^{45}$ was applied to optimize the generated model by making re-contribution of the weight to the lattice points. As a region focusing procedure, Discriminant power was used.

Partial Least-Square (PLS) Analysis and Validation. Partial Least Squares Analysis was performed by the QSAR module in SYBYL to build a linear relationship between dependant 
and independent variables, which are the $\mathrm{pIC}_{50}$ value and the CoMSIA descriptors, respectively. The optimal number of components was determined by considering a $Q^{2}$ value, which was generated by the leave-one-out (LOO) cross-validation method using SAMPLS. ${ }^{46}$ The number of components was increased until additional compounds did not increase $Q^{2}$ by at least $5 \%$ per added component. A non-cross-validated analysis was carried out using this optimal number of components. PRESS (the root mean predictive error sum of square), $F$ value, and standard error of estimate (SEE) value were calculated along with the non-cross-validated-correlation coefficient. Column filtering value was set to $2.0 \mathrm{kcal} / \mathrm{mol}$ to increase the signal to noise raton when PLS analysis. The equation for the crossvalidated coefficient is as follows:

$$
r_{c v}^{2}=1-\frac{\sum\left(Y_{\text {predicted }}-Y_{\text {observed }}\right)^{2}}{\sum\left(Y_{\text {observed }}-Y_{\text {mean }}\right)^{2}}
$$

where $\mathrm{Y}_{\text {predicted }}, \mathrm{Y}_{\text {observed }}$, and $\mathrm{Y}_{\text {mean }}$ are the predicted, observed, and mean values of the target property $\left(\mathrm{pIC}_{50}\right)$, respectively. $\sum\left(Y_{\text {predicted }}-Y_{\text {observed }}\right)^{2}$ is the predictive sum of squares.

To validate the derived QSAR model, bootstrapping and crossvalidation were carried out. The basic idea of bootstrapping is that it simulates a statistical sampling procedure randomly generating many new data sets from the original data set, and the parameter is calculated between original data set and the average of many bootstrap samplings to measure the bias of the original calculation. In the case of crossvalidation, leaveone-out (LOO) and leave-group-out (five groups and ten groups) were performed to validate the model. The concept of crossvalidation is that some molecules are omitted from the data set, and then a model is generated by compounds left, which will predict the activity of the omitted compounds. Leave-oneout will exclude one compound in each running, and generate the model using remaining compounds to predict the excluded one. In the case of group crossvalidation of five, $4 / 5$ ths of all compounds will generate the model, and predict the remaining $1 / 5$ th of all compounds by the generated model. The predictive ability of the model was evaluated by the predictive correlation coefficient, $r_{\text {pred }}^{2}$. A total of 11 compounds, which were not included in the training set, were predicted by the model, and $r_{\text {pred }}^{2}$ was calculated using following equation:

$$
r_{\text {pred }}^{2}=1-(\mathrm{PRESS} / \mathrm{SD})
$$

where SD is the sum of the squared deviation between the actual activities of the compounds in the test set and the mean activity of the compounds in the training set, and PRESS is the sum of the squared deviations between predicted and actual activities for every compounds in the test set.

Binding Free Energy Calculation. To validate using the geometry of docked pose of the most active compound, we calculated the binding free energy of the aligned compounds. It was calculated by the linear interaction energy (LIE) method. LIE method assumes that the binding energy can be simply calculated by considering the energy of the bound complex with the energy of the free ligand-receptor system. Liaison ${ }^{47}$ implemented in Schrödinger package predict ligand-protein binding free energies using the Surface Generalized Born (SGB) continuum model for solvation. Contrary to the explicit-solvent method of Åqvist, ${ }^{48}$ Liaison performs calculations based on the implicit solvent, which improves the computational efficiency. ${ }^{47}$ The $\Delta G_{b i n d}$ is derived by the following empirical equation :

$$
\begin{aligned}
\Delta G_{\text {bind }}= & \alpha\left(<\mathrm{U}_{v d w}^{b}>-<\mathrm{U}_{v d w}^{f}>\right)+\beta\left(<U_{\text {elec }}^{b}>\right. \\
& \left.-<\mathrm{U}_{\text {elec }}^{f}>\right)+\gamma\left(<\mathrm{U}_{c a v}^{b}>-<\mathrm{U}_{c a v}^{f}>\right),
\end{aligned}
$$

where $\alpha, \beta$ and $\gamma$ are the coefficients ; $\mathrm{b}$ indicates the bound form of the ligand; $\mathrm{f}$ indicates the free form of the ligand $;<>$ represents the ensemble average ; $\mathrm{U}_{v d w}$ represents the van der Waals interaction $\mathrm{U}_{\text {elec }}$ represents the electrostatic contribution; $\mathrm{U}_{\text {cav }}$ represents energy term proportional to the exposed surface area of the ligand in SGB continuum solvent model. ${ }^{49}$ The Liason's simulation calculates the values of $\mathrm{U}_{v d w}, \mathrm{U}_{\text {elec }}$, and $\mathrm{U}_{c a v}$ of training-set compounds. Using those LIE-SGB descriptors $\left(\mathrm{U}_{v d w}, \mathrm{U}_{\text {elec }}\right.$, and $\left.\mathrm{U}_{c a v}\right)$, Multiple Linear Regression was carried out to predict $\mathrm{pIC}_{50}$ value of compounds. The experimental $\mathrm{pIC}_{50}$ of the hERG blocking activity was used as a dependant variable when building the model. Strike ${ }^{50}$ implemented in the Schrödinger package was used for those statistical calculations.

\section{hERG Binding Assay.}

Cell Culture: CHO-K1 cells expressing hERG (human ethera-go-go-related gene) channels via the inducible Tet-On gene expression system (CHO-K1 Tet-On hERG cells) were purchased from IonGate Biosciences GmbH (Frankfurt, Germany). CHO-K1 Tet-On hERG cells were cultured in Dulbecco's modified Eagle's medium containing $10 \%(\mathrm{v} / \mathrm{v})$ fetal bovine serum, penicillin (100 U/mL), streptomycin $(100 \mu \mathrm{g} / \mathrm{mL}), \mathrm{G} 418$ (200 $\mu \mathrm{g} / \mathrm{mL})$, hygromycin $(200 \mu \mathrm{g} / \mathrm{mL})$, puromycin $(2 \mu \mathrm{g} / \mathrm{mL})$, and fungizone $(2.5 \mu \mathrm{g} / \mathrm{mL})$ in humidified $5 \% \mathrm{CO}_{2}$ at $37^{\circ} \mathrm{C}$. Cells were passaged every three days by treatment with trypsin. For hERG channel expression, the Tet-On gene expression system was induced by adding $5 \mu \mathrm{g} / \mathrm{mL}$ of doxycyclin (Sigma, St. Louis, MO, USA) into the growth medium. Approximately 20 to $32 \mathrm{~h}$ after addition of doxycyclin, the cells were used for whole-cell recordings.

Electrophysiology. Micropipettes were pulled from borosilicate glass capillaries (GC150T-7.5 Warner Instrument Corp., Hamden, CT, USA) on a puller (P-97 Sutter Instrument Co., Novato, CA, USA) with resistance of typically $\sim 3$ - $4 \mathrm{M}$. Whole-cell currents were recorded with the pipette (intracellular) solution containing (in mM): $130 \mathrm{KCl}, 1 \mathrm{MgCl}_{2}$, 5 EGTA, $5 \mathrm{Mg}$-ATP, and 10 HEPES, pH to 7.2 and with the bath (extracellular) solution containing (in $\mathrm{mM}$ ): $137 \mathrm{NaCl}, 4 \mathrm{KCl}, 10$ glucose, 1.8 $\mathrm{CaCl}_{2}, 1 \mathrm{MgCl}_{2}$, and $10 \mathrm{HEPES}, \mathrm{pH}$ to 7.4. Each test compound was dissolved in DMSO as $100 \mathrm{mM}$ stock solutions, and was diluted in the bath solution and directly perfused to the bath. Whole-cell recordings were performed at room temperature with an EPC-10 patch clamp amplifier (HEKA, Germany) and were low-pass filtered $(5 \mathrm{kHz})$ with a 4-pole Bessel filter. $\mathrm{hERG}$ channel tail currents were evoked by repolarizing steps to -50 $\mathrm{mV}$ for $500 \mathrm{~ms}$ following a 200-ms depolarization potential of $20 \mathrm{mV}$ at a holding potential of $-80 \mathrm{mV}$ with 20 -s sweep intervals. Whole-cell currents were acquired and digitized at 10 
kHz using the PATCHMASTER (HEKA, Germany).

Data Analysis. Whole-cell recordings were analyzed using the PATCHMASTER/FITMASTER (HEKA, Germany) and the GraphPad Prism 4 (GraphPad Software, Inc., La Jolla, CA, USA)software. The dose-response curve was fitted to a Hill equation (sigmoidal dose-response equation), $I=I_{\min }+\left(I_{\max }-\right.$ $\left.I_{\min }\right) /\left(1+10^{\left(\left(\operatorname{LogIC} C_{50}-C\right) \times h\right)}\right)$ where $I_{\min }$ and $I_{\max }$ are the normalized minimum and maximum tail currents, respectively. $\mathrm{IC}_{50}$ is the half-maximum inhibition concentration. $C$ is the logarithm of concentration. $h$ is the hill coefficient. All results are presented as the means \pm S.E.M.

\section{Results and Discussion}

Three different CoMSIA models were generated by different alignment approaches using 38 T-type calcium channel blockers. In CoMSIA, the alignment based on bioactive conformation will guarantee a reliable model. Unfortunately, no X-ray data of hERG blockers has been disclosed so far. Therefore, it was needed to generate a bioactive conformer of the compound. Due to its high flexibility of the compounds, we adopted a different alignment approach to identify the bioactive conformation. The conformation was changed according to a different alignment approach. Three different conformations of the most active compound are shown in Fig. 1.

CoMSIA Statistical Results. Three different CoMSIA models were generated based on different alignment methods. The different conformations of the most active compounds were superimposed, and presented in Fig. 2. This demonstrates that the flexibility of the template molecule is high, and that more careful determination about bioactive conformation is needed.

The first model based on the global minimized conformation of the most active compound (Fig. 2A) gave the $q^{2}$ of 0.521 with an optimal number of components of three and a non-crossvalidated correlation coefficient $r^{2}$ of $0.790\left(q^{2}>0.5\right)$ (Table 3 ). In contrast to CoMFA, CoMSIA has five different fields, and it is highly unlikely that they are independent of each other. ${ }^{51}$ Such dependencies of the individual fields usually decrease the signal-to-noise ratio in the data, ${ }^{52}$ and lower the statistical significance of the results. ${ }^{51}$ Therefore, 31 possible combinations of different fields were carried out to obtain the best CoMSIA model (data not shown). The steric, electrostatic, hydrophobic, and H-bond donor fields yielded the best results.

The second method for the alignment was based on the pharmacophore of ligands (Fig. 2B). The program PHASE (Pharmacophore Alignment and Scoring Engine), which we used for our compound alignment, generates reasonable conformations, and finds plausible pharmacophores with its tree-based partitioning algorithm, which will be evaluated according to the scoring function and also by building a PLS regression model. ${ }^{53} \mathrm{We}$ adjusted the number of sites (the number of pharmacophores) as six, because five sites generated a too small number of pharmacophore hypotheses, and seven sites yielded a too large number of pharmacophore hypotheses. Those six pharmacophores were comprised of two hydrogen bond acceptors (A), two aromatic rings (R), one hydrophobic group $(\mathrm{H})$, and one positively charged group (P). A total of 1060 hypotheses were obtained by those six features pharmacophores. These hypotheses were scored
(A)

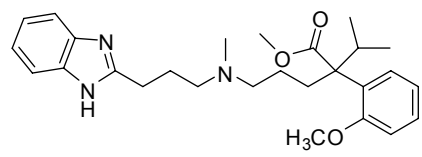

(B)

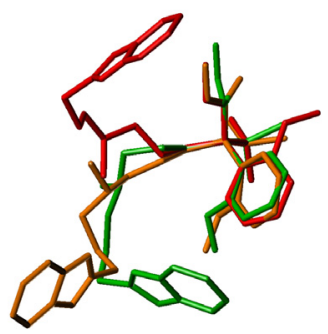

Figure 1. Three different conformations of the most active compound. (A) structural drawing of the most active compound; (B) superimposition of three different conformations of the most active compound. 'Fit Atoms' was used to superimpose the structures. Green denotes the global minimized conformation, orange denotes the pharmacophore based conformation and red denotes the receptor guided conformation.
(A)

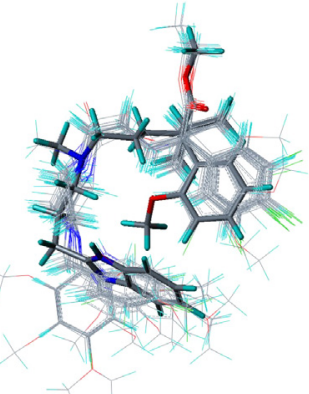

(C)

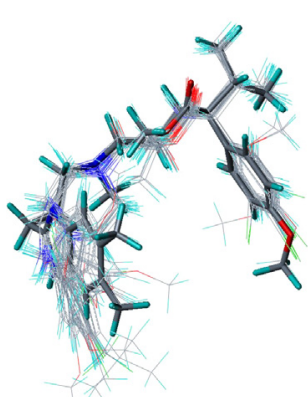

(B)

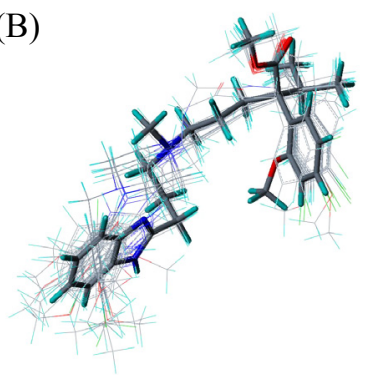

Figure 2. Alignment of the training and test sets: (A) ligand based alignment, (B) pharmacophore based alignment and (C) receptor guided alignment. (A template molecule is shown as a stick model.)

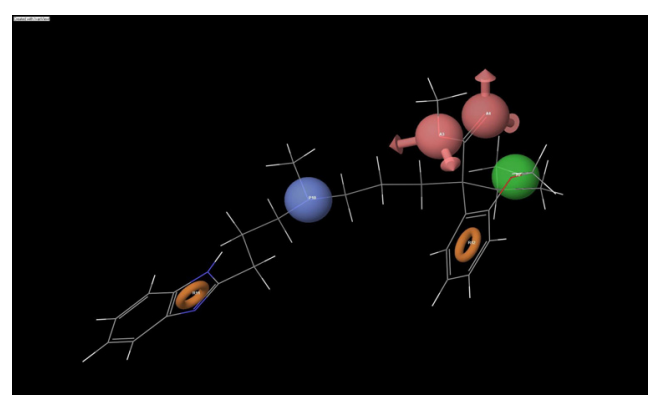

Figure 3. Mapping of the Common Pharmacophore Hypothesis (ID = 15 ) with the most active compound. Pink transparent spheres with arrows, a blue sphere, a green sphere, and orange toroids represent $\mathrm{H}$ bond acceptors, a negatively charged group, a hydrophobic group, and aromatic rings, respectively. 
by a set of active compounds with a $\mathrm{pIC}_{50}$ value greater than 6 , and 54 highly ranked hypotheses were retained. Using each hypothesis, we tried building a 3D QSAR model by partial leastsquares regression. Out of the 54 hypotheses, 13 hypothesesshowed greater than 0.5 of $q^{2}$. We selected the hypothesis that showed the highest $q^{2}$ value, and employed those alignments to perform the CoMSIA analysis. However, the result did not show good CoMSIA statistics. Thus, we exported each alignment of compounds by each pharmacophore hypothesis to SYBYL, and

Table 3. Statistical data for CoMSIA models using different alignment methods

\begin{tabular}{lccc}
\hline \multirow{2}{*}{ Component } & \multicolumn{3}{c}{ CoMSIA } \\
\cline { 2 - 4 } & Alignment 1 & Alignment 2 & Alignment 3 \\
\hline$q^{2 a}$ & 0.521 & 0.572 & 0.781 \\
$r^{2 b}$ & 0.790 & 0.912 & 0.955 \\
$r_{\text {pred }}^{2}{ }^{2}$ & 0.745 & 0.501 & 0.758 \\
$r_{\text {boot }}^{2}{ }^{e}$ & 0.824 & 0.947 & 0.964 \\
$r_{5 C V}^{2}{ }^{f}$ & 0.375 & 0.527 & 0.747 \\
$r_{10 C V}^{2}$ & 0.484 & 0.542 & 0.702 \\
PLS components $^{g}$ & 3 & 5 & 6 \\
F value $^{h}$ & 32.589 & 49.751 & 82.291 \\
SEE $^{i}$ & 0.195 & 0.131 & 0.095 \\
Field Contribution: & & & \\
Steric & 0.043 & 0.159 & \\
Electrostatic & 0.442 & 0.552 & 0.196 \\
Hydrophobic & 0.196 & 0.289 & 0.243 \\
Donor & 0.320 & & 0.334 \\
Acceptor & & & 0.228 \\
\hline
\end{tabular}

${ }^{a}$ correlation coefficient of leave-one-out cross validation. ${ }^{b}$ non-crossvalidated correlation coefficient. ${ }^{c}$ predictive correlation coefficient to the test set. ${ }^{d}$ average of correlation coefficient after 100 bootstrapped run. ${ }^{e}$ Average cross-validated correlation coefficient for 10 runs using 5 cross-validation groups. ${ }^{f}$ Average cross-validated correlation coefficient for 10 runs using 10 cross-validation groups. ${ }^{g}$ Optimal number of principal components. ${ }^{h}$ Ratio of $r^{2}$ explained to unexplained $=r^{2} /\left(1-r^{2}\right) .{ }^{i}$ Standard error of estimate. carried out the CoMSIA PLS calculation, one by one. Finally, the best CoMSIA PLS result was obtained from the alignment by the pharmacophore hypothesis ID number 15 (Fig. 3), which also gave a perfect fitness score (3.0) to the most active compound. This model showed a $q^{2}$ of 0.572 and a $r^{2}$ of 0.912 in CoMSIA statistics (Table 3). The highest $q^{2}$ was obtained by the combination of steric, electrostatic, and hydrophobic fields.

When ligands bind to a receptor, the global minimum conformation may not be the best conformation. This is because ligands need some degree of torsional freedom to accommodate themselves to the receptor-binding site for lowering the energy of a drug-receptor complex. ${ }^{54}$ For that phenomenon, docked conformation of structure also has been employed when aligning compounds in the 3D QSAR. Besides, studies have shown that receptor-guided alignment produced better results than those from a ligand-based approach due to its more realistic information about the receptors. ${ }^{55,56}$ Therefore, we adopted a receptorguided alignment by employing the docked conformation of the most active compound (Fig. 2C). The docking pose of compound 2 by Glide XP was shown in Fig. 4 and its Gscore value was -8.28 . Third CoMSIA model was derived based on this conformation. The model shows that its $q^{2}$ and $r^{2}$ were 0.781 and 0.955 , respectively. Carrying out 31 possible combinations of descriptors (Table 3, 4), electrostatic, hydrophobic, H-bond donor, and acceptor fields showed the best result.

We applied the linear interaction energy (LIE) method to predict the binding affinity of aligned compounds generated by the receptor guided alignment for the sake of the validation of this approach. After generating the model by multiple linear regression, we detected three compounds (compound 9, 15, and 26) presenting large residuals. Two compounds out of them showed greater than $6 \mu \mathrm{M}$ of $\mathrm{IC}_{50}$ value $\left(\mathrm{IC}_{50}\right.$ of compound $9=$ $6.58 \mu \mathrm{M}$ and $\mathrm{IC}_{50}$ of compound $26=5.17 \mu \mathrm{M}$ ), which was relatively lower activity than that of others, and remaining compound also showed greater than $4 \mu \mathrm{M}$ of $\mathrm{IC}_{50}$ value $\left(\mathrm{IC}_{50}\right.$ of compound $15=4.3 \mu \mathrm{M})$. It was assumed that three compounds did not follow the docked pose of the most active compound due to its low activity. Removal of these three compounds resulted in a model with $r^{2}$ of 0.5131 and leave-one-out crossvalidated

Table 4. Summary of the PLS analyses by receptor guided alignment using different CoMSIA field combinations

\begin{tabular}{|c|c|c|c|c|c|c|c|c|c|c|}
\hline \multirow{2}{*}{ CoMSIA field $^{a}$} & \multirow{2}{*}{$q^{2}$} & \multirow{2}{*}{$\mathrm{N}^{b}$} & \multirow{2}{*}{$r^{2}$} & \multirow{2}{*}{ SEE } & \multirow{2}{*}{ F value } & \multicolumn{5}{|c|}{ contributions } \\
\hline & & & & & & $\mathrm{S}$ & E & $\mathrm{H}$ & $\mathrm{D}$ & A \\
\hline $\mathrm{S}$ & -0.024 & 1 & 0.164 & 0.374 & 5.505 & 1 & & & & \\
\hline $\mathrm{E}$ & -0.118 & 1 & 0.078 & 0.393 & 2.358 & & 1 & & & \\
\hline $\mathrm{H}$ & 0.127 & 1 & 0.339 & 0.333 & 14.379 & & & 1 & & \\
\hline $\mathrm{D}$ & 0.104 & 1 & 0.275 & 0.349 & 10.600 & & & & 1 & \\
\hline A & 0.102 & 2 & 0.378 & 0.329 & 8.203 & & & & & 1 \\
\hline SEHD & 0.157 & 5 & 0.783 & 0.206 & 17.303 & 0.084 & 0.297 & 0.343 & & \\
\hline SEHA & 0.144 & 5 & 0.835 & 0.180 & 24.205 & 0.137 & 0.262 & 0.366 & & 0.235 \\
\hline SEDA & 0.690 & 7 & 0.918 & 0.132 & 35.248 & 0.149 & 0.312 & & 0.382 & 0.290 \\
\hline SHDA & 0.744 & 5 & 0.947 & 0.102 & 86.145 & 0.097 & & 0.231 & 0.382 & 0.290 \\
\hline EHDA & 0.781 & 6 & 0.955 & 0.095 & 82.291 & & 0.196 & 0.243 & 0.334 & 0.228 \\
\hline SEHDA & 0.721 & 6 & 0.953 & 0.098 & 77.809 & 0.077 & 0.183 & 0.211 & 0.316 & 0.213 \\
\hline
\end{tabular}

${ }^{a}$ Abbreviations: S(steric), E (electrostatic), H (hydrophobic), D (hydrogen bond donor), A (hydrogen bond acceptor) ${ }^{b}$ Number of principal components. 
$r^{2}$ of 0.3435 . Although the obtained statistical result is quite lower than that of the CoMSIA models, the $q^{2}$ of greater than 0.3 with $r^{2}$ of greater than 0.5 is also considered a meaningful correlation between dependant and independent variables. ${ }^{57,58}$ Therefore, this calculation suggests that there is linear relationship between $\mathrm{pIC}_{50}$ of experimental hERG blocking activity and calculated binding interaction energy from the geometry of aligned compounds based on the most active compound and insures reliable process of receptor guided alignment. Fig. 6 shows the linear relationship between the predicted and experimental $\mathrm{pIC}_{50}$ by the LIE method

Validation of CoMSIA Model \& Model Selection. The predictive $r^{2}$ of external test set, bootstrapped $r^{2}$, and leave-groupout crossvalidated $r^{2}$ were determined for validating the CoMSIA model. Eight compounds were selected for an external test set, and those were minimized, and aligned by a method similar to that of the training set. Then, activities were predicted by each CoMSIA model. The selected external test set yielded a $r_{\text {pred }}^{2}$ of $0.745,0.501$ and 0.758 by alignment 1,2 , and 3 , respectively, which implies that alignment 3 has the best external predictive power. In the case of $r_{\text {boot }}^{2}$, alignment 3 also showed the highest value, 0.964 , followed by 0.947 for alignment 2 and 0.824 for alignment 1 . Further validation was carried out using leave-group-out crossvalidation. After ten running of leave-10\%-out and leave- $20 \%$-out crossvalidation, the mean value by alignment 3 was found which was more stable and robust than those of any other alignments: $r_{10 \mathrm{CV}}^{2}=0.702$ and $r_{5 \mathrm{CV}}^{2}=$ 0.747 . The results by other alignment methods are also summarized in Table 3.

Since the CoMSIA model by alignment 3 showed the best result in all validation criteria, indicating good internal prediction as well as external predictive power of the model, we selected this model for the analysis of the CoMSIA contour map. Fig. 5C depicts the graph of the experimental versus predicted activities for the training and test set of molecules by alignment 3 , and Table 4 shows the statistics of the combination of different fields to find the best CoMSIA model by alignment 3 .

CoMSIA Contour Map. The hydrophobic, H-bond donor \& acceptor, and the electrostatic fields were generated by the best CoMSIA model, which is obtained by alignment 3. Each

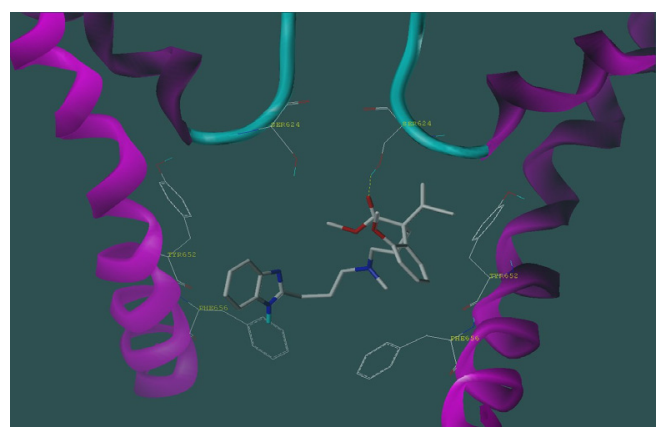

Figure 4. Docked structure of the most active compound into the hERG channel pore of homology model. The most active compound was represented by stick format ; residues involved in hydrogen bonding interaction (Ser 624) and hydrophobic interaction (Tyr 652, Phe 656) were displayed using a line format. For the sake of clarity, only two monomers (B, D) among the tetramers were displayed. field was analyzed to find out the correlation between the activity trend and the structural modification. The contour map of all four fields is shown in Fig. 7, and the respective contour maps of the fields are shown in Fig. 8. Since the best model was obtained by alignment 3 , which employed the ligand binding site of the hERG receptor to generate the bioactive conformer, it is also worth while comparing those binding site with the CoMSIA
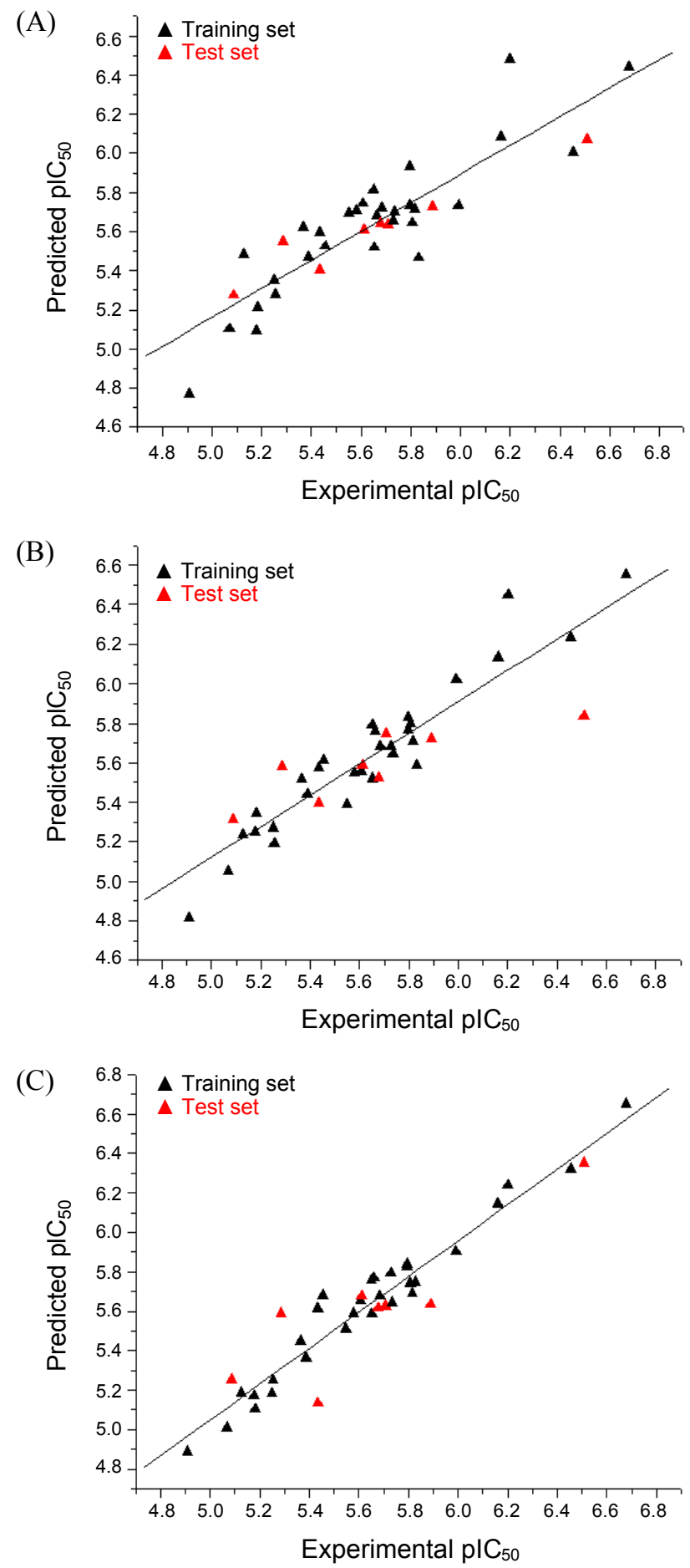

Figure 5. Plots of the predicted versus experimental $\mathrm{pIC}_{50}$ data of the 3D-QSAR from the CoMSIA model for the training and test sets; (A) ligand based alignment, (B) pharmacophore based alignment and (C) receptor guided alignment 
contour. The CoMSIA contour map was superimposed on the lipophilic potential map of the hERG channel cavity. This showed an explainable matching each other and indicated usefulness of receptor guided alignment. The lipophilic potential was generated, and displayed using the MOLCAD module in SYBYL (Fig. 9).

Hydrophobic Field. The contour map of the hydrophobic field for the model by alignment 3 is depicted in Fig. 8A. The contours in yellow and white (not shown) indicate regions where hydrophobic favorable groups increase and decrease activity, respectively. One large yellow contour in the 4,5-position of $\mathrm{R}_{1}$ phenyl ring represents the region where the hydrophobic group, increases its activity. Therefore, the presence of the halogen group, which is hydrophobic, resulted in an increased activity. For example, compounds $6\left(\mathrm{pIC}_{50}=6.4559\right), 7\left(\mathrm{pIC}_{50}=6.5086\right)$, and $8\left(\mathrm{pIC}_{50}=5.6498\right)$ with a $-\mathrm{F}$ in the 4 position of the $\mathrm{R}_{1}$ phenyl ring have much higher activity than compound $9\left(\mathrm{pIC}_{50}=5.1818\right)$ with a $-\mathrm{Br}$ in the 3 position of the $\mathrm{R}_{1}$ phenyl ring in benzimidazole derivatives. Compound $7\left(\mathrm{pIC}_{50}=6.5086\right)$ with a $-\mathrm{F}$ in the 4 position also shows higher activity than compound $4\left(\mathrm{pIC}_{50}=\right.$ 6.2006) with no substituent in the 4,5-position and a $-\mathrm{OCH}_{3}$ group in the 6-position. There are also some compounds in piperazine derivatives following this trend. In the case of compounds with high activity (e.g., compounds 27-36), they have a fluorine atom at the 4-position of the $\mathrm{R}_{1}$ phenyl ring, while less active compounds (e.g., compound 21-24) have no substituent at the 4,5-position of the $\mathrm{R}_{1}$ phenyl ring ; these compounds only have a bromine atom at the 3 -position of $\mathrm{R}_{1}$ phenyl ring. Another example is shown when comparing compound $18\left(\mathrm{pIC}_{50}=\right.$ 5.8297 ) having a $-\mathrm{Br}$ in the 4 position with corresponding compound $23\left(\mathrm{pIC}_{50}=5.0878\right)$ having a $-\mathrm{Br}$ in the 3 position. Sometimes, it is observed that the activity deteriorated due to the oxygen in the hydrophilic methoxy substituent falling into this hydrophobic-favorable region. For example, considering benzimidazole derivatives, compound $1\left(\mathrm{pIC}_{50}=5.5482\right)$ bearing methoxy group at the 4-position of the $\mathrm{R}_{1}$ phenyl ring has much less activity than compounds $2\left(\mathrm{pIC}_{50}=6.6778\right)$ and $4\left(\mathrm{pIC}_{50}=6.2006\right)$ bearing the methoxy group at the 6-position, which is outside of the yellow contour. Compound 5 having methoxy at the 3,4-position also showsquite less activity than compound 2 and 4 , which we can explain by above trend. This hydrophobicfavorable contour also shows the consistency with the 3D topology of the receptor. It is observed that the yellow polyhedron is hanging on the lipophilic potential map of Tyr 652 and Phe 656 (Fig. 9). These two residues are known as critical hydrophobic residues for hERG blockade. ${ }^{5}$ Therefore, the hydrophobic substituents falling this region will also have chances to interact with these residues through hydrophobic interaction or $\pi-\pi$ interaction, which is known as important drug binding interaction in hERG channel. ${ }^{59}$

H-bond Donor \& Acceptor Field. The H-bond donor and acceptor contour maps are shown in Fig. 8B. Contours in magenta and red (not shown) represent the H-bond acceptor favorable and unfavorable regions, while the cyan and purple contours depict the place where the H-bond donors are favorable and unfavorable, respectively. One big magenta-colored polyhedron and puple colored contour, which almost overlaps with the magenta polyhedron, are located just above the structure. It indi-

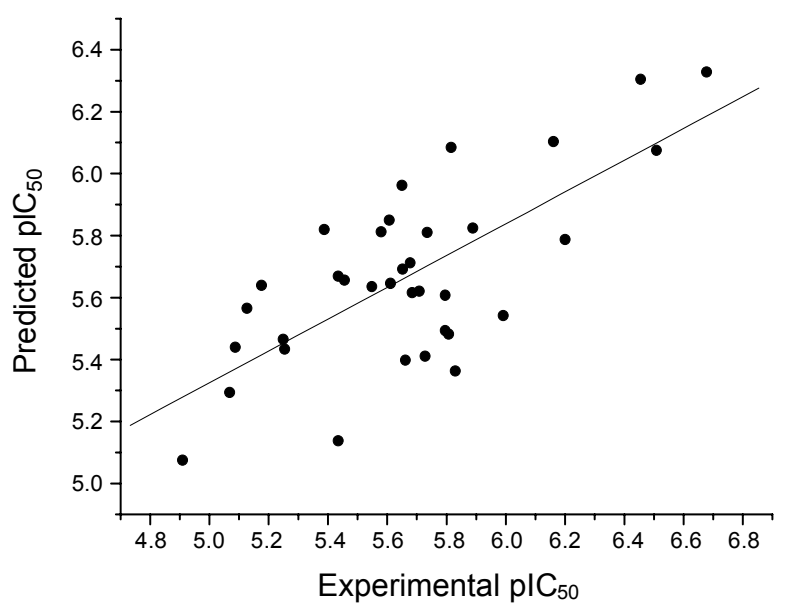

Figure 6. Plot of the experimental versus predicted $\mathrm{pIC}_{50}$ by multiple linear regression using LIE-SGB descriptors.

cates that the H-bond acceptor moiety near this contour map in the structure leads to higher activity of compounds, while the $\mathrm{H}$-bond donor moiety near this contour map in the structure causes lower activity of compounds. This hypothesis is supported by the fact that the nitrogen atom of the benzimidazole moiety as the $\mathrm{H}$-bond acceptor, oxygen atom in the $\mathrm{CO}_{2} \mathrm{Me}$ moiety, and the NH moiety in the linker chain near the magenta contour affects the inhibitory potency trend. For example, all of the compounds having an inhibitory potency value below $1 \mu \mathrm{M}$ of $\mathrm{IC}_{50}$ value are benzimidazole derivatives (compounds 2, 4-7) and four out of the remaining six benzimidazole derivatives also show inhibitory potency value below $3 \mu \mathrm{M}$ of $\mathrm{IC}_{50}$ value : compound $1\left(\mathrm{IC}_{50}=2.83 \mu \mathrm{M}\right)$ compound $3\left(\mathrm{IC}_{50}=2.47 \mu \mathrm{M}\right)$ compound $8\left(\mathrm{IC}_{50}=2.24 \mu \mathrm{M}\right)$, and compound $14\left(\mathrm{IC}_{50}=1.6 \mu \mathrm{M}\right)$. Furthermore, comparing compound 11 with 38, both of which have a different scaffold compared to others- having NHCO linker and no $\mathrm{CO}_{2} \mathrm{Me}$ moiety-it is clear that compounds having a benzimidazole moiety is also of higher potency to hERG channel than compounds with a piperazine moiety. Only two compounds among benzimidazole derivatives have greater than $5 \mu \mathrm{M}$ of $\mathrm{IC}_{50}$ value. Compound $9\left(\mathrm{IC}_{50}=5.1818 \mu \mathrm{M}\right)$ out of these two compounds have a benzoxazole moiety instead of a benzimidazole moiety. There is oxygen atom instead of nitrogen in benzoxazole, which resulted in the devoid of H-bond donor. We can observe that there is a small cyan contour located near this moiety representing the $\mathrm{H}$-bond donor-favorable region. Therefore, it is explainable that the compound 9 has a relatively lower inhibitory potency. Other structural elements that contribute to the formation of the magenta colored as well as the purple colored contours are the oxygen atom in the $\mathrm{CO}_{2} \mathrm{Me}$ moiety and nitrogen atom, which can act as an $\mathrm{H}$-bond acceptor and donor in the NH linker region, respectively. This is supported by the following example. Comparing compound 38 with compound 17 , it is clear that compound 38 -having a no $\mathrm{CO}_{2} \mathrm{Me}$ moiety and having a NHCO linker moiety-is less active. This is because compound 38 does not have oxygen as an H-bond acceptor near the magenta contour, and even has a nitrogen atom as an H-bond donor near the purple contour. Last but not the least, these two H-bond related fields show correlations with 


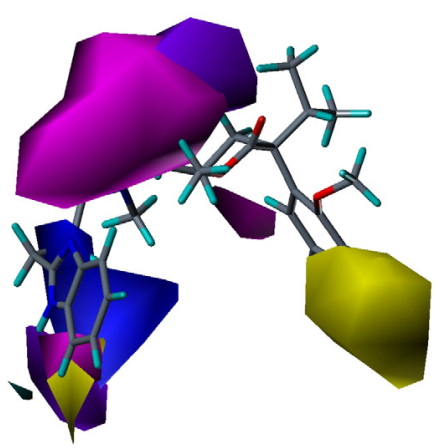

Figure 7. CoMSIA contour map (standard deviation $\times$ coefficient) of all four fields with the template compound. The yellow and white (not shown) ( $80 \%$ and $20 \%$ contributions) contours indicates hydrophobic favorable and unfavorable regions, the magenta and red (not shown) ( $80 \%$ and $20 \%$ contributions) contours represent H-bond acceptor favorable and unfavorable regions, the cyan and purple contours depict $\mathrm{H}$-bond donor favorable and unfavorable regions, while red and blue contours depict electronegative and electropositive regions.
(A)
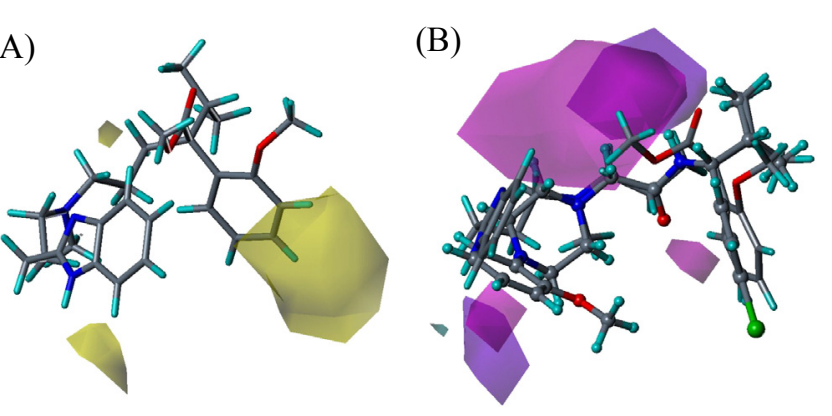

(C)

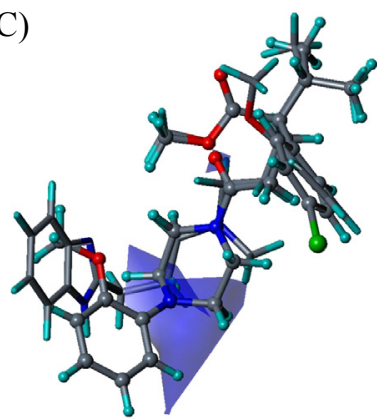

(B)

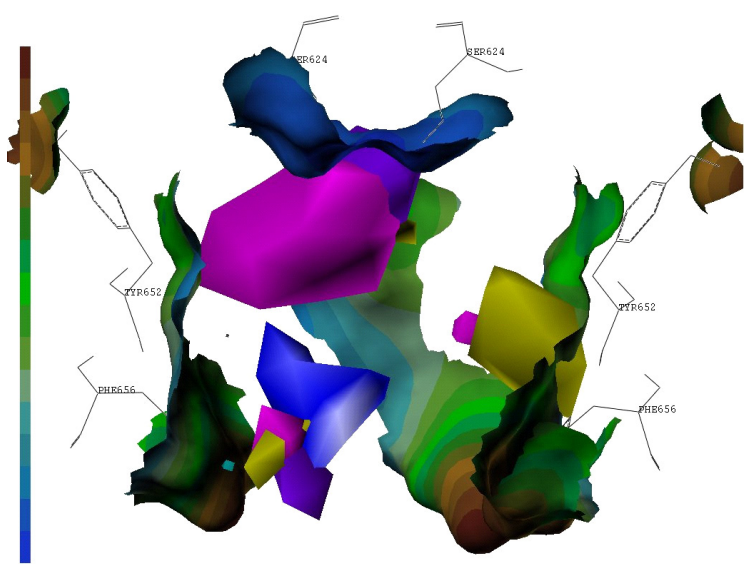

Figure 9. The CoMSIA contour maps generated by the Receptor guided alignment within the hERG channel pore (MOLCAD surfaces were generated around the hERG channel by the Fast Connolly type and Map Property as a Lipophilic Potential was calculated on that surface. The color brown represents the most lipophilic region and the color blue represents the most hydrophilic regions. For the sake of clarity, only the residues involved in hydrogen bonding interaction and hydrophobic interaction of monomer B and D were displayed).
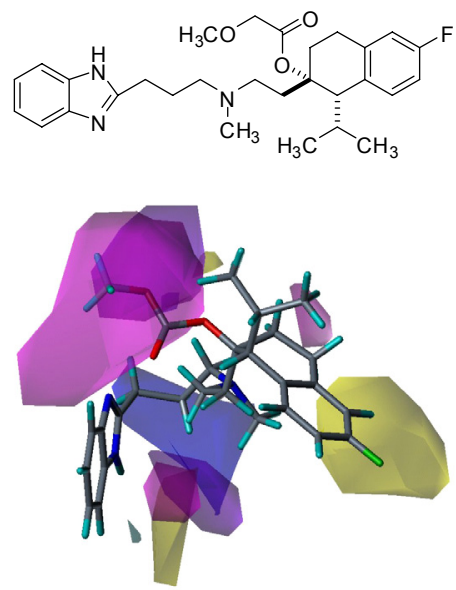

Figure 10. (A) the Structure of Mibefradil (B) Mibefradil superimposed to the CoMSIA contour plot of the best model

Figure 8. CoMSIA contour map (standard deviation $\times$ coefficient): (A) hydrophobic field for compound 2 (yellow ( $80 \%$ contribution): hydrophobic favorable; white (not shown, 20\% contribution): hydrophobic unfavorable; (B) H-bond donor and acceptor field for compound 2 illustrated as a capped stick form, compound 38 illustrated as a ball \& stick form (magenta ( $80 \%$ contribution): H-bond acceptor favorable; red ( $20 \%$, not shown): H-bond acceptor unfavorable; cyan $(80 \%$ contribution): H-bond donor favorable; purple ( $20 \%$ contribution): H-bond donor unfavorable); (C) Electrostatic field for compound 2 colored in yellow, compound 29 colored in atom type (blue ( $80 \%$ contribution): negative potential unfavorable; red ( $20 \%$ contribution): negative potential favorable)

the lipophilic potential map of the hERG channel. The magenta and purple colored contours which appear toward the blue lipophilic potential region indicate the existence of a polar region in the $h E R G$ channel pore, which is characterized by polar residues Ser 624 near the pore helix. ${ }^{1}$ A site-directed mutagenesis approach revealed that Ser 624 was also a key residue in the hERG- ligand binding, and that this residue interacted with the polar regions of many hERG blockers. ${ }^{1,4,10,60,61}$ Therefore, the CoMSIA H-bond acceptor and donor fields suggest a good consistency for those biological factors.

Electrostatic Field. The electrostatic field contour is presented in Fig. 8C. The blue contour represents the regions where the positive potential is favorable to activity, while the red (not shown) depicts the negative potential favorable site. It is observed that one blue colored three dimentional isopleth is distributed from the nitrogen of the piperazine moiety to the 6position of the $\mathrm{N}$-phenyl ring in the $\mathrm{R}_{5}$ substituent. Therefore, this contour can explain the fact that any electronegative substituents in the 6-position of the N-phenyl ring in the relevant compounds can make the inhibitory potency less than their corresponding unsubstituted or electropositive substituted analogues at this position. For example, the introduction of $\mathrm{F}$ 
$\left(\mathrm{pIC}_{50}=5.2856\right)$ and $\mathrm{OCH}_{3}\left(\mathrm{pIC}_{50}=5.068\right)$ to the 6 position of the phenyl ring (compounds 26 and 38 ) gives lower inhibitory potency than the 3-methoxy and 4-methoxy substituted compounds $29\left(\mathrm{pIC}_{50}=5.7959\right)$ and $30\left(\mathrm{pIC}_{50}=5.8894\right)$. Another small polyhedron exists on a hydrogen atom of the $\mathrm{CH}_{2}$ linker next to a nitrogen atom of piperazine. This means that the electronegative group falling in this region will decrease the inhibitory potency. For example, it is found that compound 10 $\left(\mathrm{pIC}_{50}=5.1759\right)$ exhibits the least inhibitory potency among benzimidazole derivatives. This is because though it has a benzimidazole moiety suggesting increased inhibitory potency, the oxygen substituent falls exactly at this position. Compound 37 $\left(\mathrm{pIC}_{50}=4.909\right)$, which is found to be the least active among all data sets, also bears a oxygen atom next to a piperazine nitrogen atom.

We also predicted the hERG blocking activity of Mibefradil. The predicted $\mathrm{pIC}_{50}$ value and corresponding residual were 5.980 and 0.135 , respectively. The selected model resulted in predicted $\mathrm{pIC}_{50}$ value that slightly deviated from the experimental $\mathrm{pIC}_{50}\left(\mathrm{pIC}_{50}=5.845\right)$, but the difference is statistically acceptable when considering the standard error of estimate of the external test set, which is 0.197 . Fig. 10B shows the structure of the Mibefradil mapped to the contour of the best CoMSIA model. Its high inhibitory potency to the hERG channel is demonstrated by the fact that the Fluoro substituent in the benzene ring of the tetrahydronaphthalene moiety falls into the yellow region where hydrophobic group increases the inhibitory potency, and the oxygen atom of methoxy in the carbonyl group is located inside the magenta colored polyhedron where the $\mathrm{H}$-bond acceptor group increases the inhibitory potency. Other two oxygen atoms in the carbonyl group are also located near this contour map. Therefore, to avoid the hERG blocking activity of this compound, those structures mentioned above need to be changed.

\section{Conclusions}

The 3D QSAR model predicting the hERG blocking activity was developed using CoMSIA methodology. Different alignment approaches were tested to find a suitable method : a ligand based alignment a pharmacophore model based alignment and a receptor guided alignment. The receptor guided alignment showed the most significant performance for all statistical values : $q^{2} r^{2} r_{\text {pred }}^{2} r_{\text {boot }}^{2} r_{5 C V}^{2}$ and $r_{10 C V}^{2}$. Subsequent binding free energy calculations further rationalized the geometry of the training and test sets from the receptor-guided alignment. The CoMSIA contour map also showed a good correlation with the lipophilic potential map of the hERG channel pore. These findings led us to conclude that the CoMSIA method with receptor-guided alignment produced more reliable output than the other two alignment methods, and was more suitable to our data sets for hERG blocking predictions. The CoMSIA descriptor fields also suggested structural requirements to mitigate the hERG blockade effect in what follows: being devoid of hydrophobic substituents at the meta and para position of the $\mathrm{R}_{1}$ phenyl ring, the carbonyl group together with the methoxy substituent bearing the oxygen atoms, which may act as $\mathrm{H}$-bond acceptors, the benzimidazole moiety bearing the nitro- gen atom, which may act as an H-bond acceptor and the electropositive substituents being located at the 6-position of the Nphenyl ring in the $\mathrm{R}_{5}$ substituent. Thus, our CoMSIA models can be used when modifying the T-type calcium channel blockers determined to be hERG blockers or when designing T-type calcium channel blockers to mitigate the hERG blockade effect at the first stage of drug development.

Acknowledgments. This study was financially supported by Korea Institute of Science and Technology.

\section{References and Notes}

1. Sanguinetti, M. C.; Martin, T. F. Nature 2006, 440, 463.

2. Warmke, J. W.; Ganetzky, B. Proc. Natl. Acad. Sci. 1994, 91, 3438.

3. Curran, M. E.; Splawski, I.; Timothy, K. W.; Vincen, G. M.; Green, E. D.; Keating, M. T. Cell. 1995, 80, 795.

4. Aronov, A. M. Drug Discov. Today 2005, 10, 149.

5. Mitcheson, J. S. Chem. Res. Toxicol. 2008, 21, 1005.

6. Long, S. B.; Campbell, E. B.; Mackinnon, R. Science 2005, 309, 897.

7. Doyle, D. A.; Morais Cabral, J.; Pfuetzner, R. A.; Kuo, A.; Gulbis, J. M.; Cohen, S. L.; Chait, B. T.; MacKinnon, R. Science 1998, 280,69

8. Jiang, Y. X.; Lee, A.; Chen, J. Y.; Cadene, M.; Chait, B. T.; MacKinnon, R. Nature 2002, 417, 523.

9. Jiang, Y.; Lee, A.; Chen, J.; Ruta, V.; Cadene, M.; Chait, B. T.; MacKinnon, R. Nature 2003, 423, 33.

10. Imai, Y. N.; Ryu, S. H.; Oiki, S. J. Med. Chem. 2009, 52, 1630.

11. Gepp, M. M.; Hutter, M. C. Bioorg. Med. Chem. 2006, 14, 5325.

12. Thai, K. M.; Ecker, G. F. Bioorg. Med. Chem. 2008, 16, 4107.

13. Yap, C. W.; Cai, C. Z.; Xue, Y.; Chen, Y. Z. Toxicol. Sci. 2004, 79, 170 .

14. Li, Q.; Jørgensen, F. S.; Oprea, T.; Brunak, S.; Taboureau, O. Mol. Pharm. 2008, 5, 117.

15. Tobita, M.; Nishikawa, T.; Nagashima, R. Bioorg. Med. Chem. Lett. 2005, 15, 2886.

16. Sun, H. ChemMedChem. 2006, 1, 315.

17. Roche, O.; Trube, G.; Zuegge, J.; Pflimlin, P.; Alanine, A.; Schneider, G. ChemBioChem. 2002, 3, 455.

18. O'Brien, S. E.; de Groot, M. J. J. Med. Chem. 2005, 48, 1287.

19. Keserü, G. M. Bioorg. Med. Chem. Lett. 2003, 13, 2773.

20. Bains, W.; Basman, A.; White, C. Prog. Biophys. Mol. Biol. 2004, 86, 205.

21. Ekins, S.; Crumb, W. J.; Sarazan, R. D.; Wikel, J. H.; Wrighton, S. A. J. Pharmacol. Exp. Ther. 2002, 301, 427.

22. Cavalli, A.; Poluzzi, E.; De Ponti, F.; Recanatini, M. J. Med. Chem. 2002, 45, 3844

23. Pearlstein, R. A.; Vaz, R. J.; Kang, J.; Chen, X.-L.; Preobrazhenskaya, M.; Shchekotikhin, A. E.; Korolev, A. M.; Lysenkova, L. N.; Miroshnikova, O. V.; Hendrix, J.; Rampe, D. Bioorg. Med. Chem. Lett. 2003, 13, 1829.

24. Cianchetta, G.; Li, Y.; Kang, J.; Rampe, D.; Fravolini, A.; Cruciani, G.; Vaz, R. J. Bioorg. Med. Chem. Lett. 2005, 15, 3637.

25. Nisius, B.; Göller, A. H. J. Chem. Inf. Model. 2009, 49, 247.

26. Klebe, G.; Abraham, U.; Mietzner, T. J. Med. Chem. 1994, 37, 4130.

27. Seierstad, M.; Agrafiotis, D. K. Chem. Bio. Drug Des. 2006, 67, 284.

28. Geelen, P.; Drolet, B.; Rail, J.; Bérubé, J.; Daleau, P.; Rousseau, G.; Cardinal, R.; O'Hara, G. E.; Turgeon, J. Circulation 2000, 102, 275.

29. Sarazan, R. D.; Crumb, W. J.; Beasley, C. M.; Emmick, J. T.; Ferguson, K. M.; Strnat, C. A.; Sausen, P. J. Eur. J. Pharmacol. 2004, $502,163$.

30. Lee, H. K.; Lee, Y. S.; Roh, E. J.; Rhim, H.; Lee, J. Y.; Shin, K. 
J. Bioorg. Med. Chem. Lett. 2008, 18, 4424.

31. SYBYL 8.1.1, Tripos Inc., 1699 South Hanley Road, St. Louis, Missouri, 63144

32. Maestro, Schrodinger, LLC, Portland, OR.

33. Barakat, M. T.; Dean, P. M. Comput.-Aided. Mol. Des. 1990, 4, 295.

34. Clark, M.; Cramer, R. D., III.; Van Opdenbosch, N. J. Comput. Chem. 1989, 10, 982.

35. Gasteiger, J.; Marsili, M. Tetrahedron 1980, 36, 3219.

36. Dixon, S. L.; Smondyrev, A. M.; Knoll, E. H.; Rao, S. N.; Shaw, D. E.; Friesner, R. A. J. Comput. Aided Mol. Des. 2006, 20, 647.

37. Luo, T.; Luo, A.; Liu, M.; Liu, X. Anesth. Analg. 2008, 106, 1161.

38. Milnes, J. T.; Witchel, H. J.; Leaney, J. L.; Leishman, D. J.; Hancox, J. C. Biochem. Biophys. Res. Commun. 2006, 351, 273.

39. Su, Z.; Chen, J.; Martin, R. L.; McDermott, J. S.; Cox, B. F.; Gopalakrishnan, M.; Gintant, G. A. Biochem. Pharmacol. 2006, 71, 278.

40. Witchel, H. J.; Dempsey, C. E.; Sessions, R. B.; Perry, M.; Milnes, J. T.; Hancox, J. C.; Mitcheson, J. S. Mol. Pharmacol. 2004, 66, 1201.

41. Scholz, E. P.; Zitron, E.; Kiesecker, C.; Lueck, S.; Kathöfer, S.; Thomas, D.; Weretka, S.; Peth, S.; Kreye, V. A.; Schoels, W.; Katus, H. A.; Kiehn, J.; Karle, C. A. Arch. Pharmacol. 2003, 368, 404.

42. Kamiya, K.; Mitcheson, J. S.; Yasui, K.; Kodama, I.; Sanguinetti, M. C. Mol. Pharmacol. 2001, 60, 244.

43. Bostrom, J.; Hogner, A.; Schmitt, S. J. Med. Chem. 2006, 49, 6716.

44. Sundriyal, S.; Bharatam, P. V. Eur. J. Med. Chem. 2009, 44, 42.

45. Datar, P.; Desai, P.; Coutinho, E.; lyer, K. J. Mol. Model. 2002, 10, 290.

46. Bush, B. L.; Nachbar, R. B., Jr. J. Comput, Aided Mol. Des. 1993, 7,587 .

47. Liaison, version 4.5, Schrödinger, LLC, New York, NY, 2007.

48. Åquist, J.; Medina, C.; Samuelsson, J.-E. Protein Eng. 1994, 7, 385.

49. Zhou, R.; Friesner, R. A.; Ghosh, A.; Rizzo, R. C.; Jorgensen, W. L.; Levy, R. M. J. Phys. Chem. B 2001, 105, 10388.

50. Strike, version 1.5, Schrödinger, LLC, New York, NY, 2005.

51. Böhm, M.; Stürzebecher, J.; Klebe, G. J. Med. Chem. 1999, 42,
458.

52. Phase, version 2.5, Schrödinger, LLC, New York, NY, User Manual 2005.

53. Norinder, U. Perspect. Drug Discovery Des. 1998, 12/13/14, 25

54. Bush, C. A.; Martin-Pastor, M.; Imberty, A. Annu. Rev. Biophys. Biomol. Struct. 1999, 28, 269.

55. Hyun, K. H.; Lee, D. Y.; Lee, B. S.; Kim, C. K. QSAR Comb. Sci. 2004, 23, 637 .

56. Kunick, C.; Lauenroth, K.; Wieking, K.; Xie, X.; Schultz, C.; Gussio, R.; Zaharevitz, D.; Leost, M.; Meijer, L.; Weber, A.; Jørgensen, F. S.; Lemcke, T. J. Med. Chem. 2004, 47, 22.

57. Clark, M.; Cramer, R. D., III.; Jones, D. M.; Patterson, D. E.; Simeroth, P. E. Tetrahedron Comput. Methods 1990, 3, 47.

58. Thomas, B. F.; Compton, D. R.; Martin, B. R.; Seamus, S. F. Mol. Pharmacol. 1991, 40, 656.

59. Fernandez, D.; Ghanta, A.; Kauffman, G. W.; Sanguinetti, M. C. J. Biol. Chem. 2004, 279, 10120.

60. Kamiya, K.; Niwa, R.; Mitcheson, J. S.; Sanguinetti, M. C. Mol. Pharmacol. 2006, 69, 1709.

61. Choe, H.; Nah, K. H.; Lee, S. N.; Lee, H. S.; Jo, S. H.; Leem, C. H.; Jang, Y. J. Biochem. Biophys. Res. Commun. 2006, 344, 72.

62. Hume, J. R. J. Pharmacol. Exp. Ther. 1985, 234, 134-140.

63. Gläser, S.; Steinbach, M.; Opitz, C.; Wruck, U. Eur. J. Heart Fail. 2001, 3, 627 .

64. PERCHENET, L.; CLEMMENT-CHOMIENNE, O. J. Pharmacol. Exp. Ther. 2000, 295, 771.

65. Chouabe, C.; Drici, M. D.; Romey, G.; Barhanin, J. Therapie. 2000, 55, 195 .

66. Zhang, S.; Zhou, Z.; Gong, Q.; Makielski, J. C.; January, C. T. Circ. Res. 1999, 84, 989.

67. Yang, T.; Snyders, D.; Roden, D. M. J. Cardiovasc. Pharmacol. 2001, 38, 737 .

68. Chouabe, C.; Drici, M. D.; Romey, G.; Barhanin, J.; Lazdunski, M. Mol. Pharmacol. 1998, 54, 695.

69. Walker, B. D.; Valenzuela, S. M.; Singleton, C. B.; Tie, H.; Bursill, J. A.; Wyse, K. R.; Qiu, M. R.; Breit, S. N.; Campbell, T. J. Br. J. Pharmacol. 1999, 127, 243.

70. Ridley, J. M.; Dooley, P. C.; Milnes, J. T.; Witchel, H. J.; Hancox, J. C. J. Mol. Cell Cardiol. 2004, 36, 701. 